\title{
A exposição do enfermeiro aos riscos ocupacionais no cuidado de pessoas com lesão de pele
}

\author{
Nurses' exposure to occupational risks in the care of people with skin lesions
}

\section{Exposición del enfermero a riesgos ocupacionales en el cuidado de personas con lesión de piel}

Kaiser, Dagmar Elaine ${ }^{1}$; da Silva, Roni Carvalho²; Duro, Carmen Lúcia Mottin³ ; da Paixão, Dilmar Xavier $^{4}$; Paz, Potiguara de Oliveira ${ }^{5}$

Como citar este artigo: Kaiser DE, da Silva RC, Duro CLM, da Paixão DX, Paz PO. A exposição do enfermeiro aos riscos ocupacionais no cuidado de pessoas com lesão de pele. J. nurs. health. 2018;8(2):e188201

\section{RESUMO}

Objetivo: identificar na produção científica nacional e internacional como ocorre a exposição do enfermeiro aos riscos ocupacionais no cuidado de pessoas com lesão de pele. Método: revisão integrativa com coleta dos dados realizada em dezembro de 2016, no Portal de Periódicos da CAPES, encontrando-se publicações nas bases PubMed, LILACS e BVS. Resultados: dos 598 artigos encontrados, foram selecionados para análise 33 publicações. 0 compilado dos artigos levou ao perfil das publicações de 2000 a 2016 e derivou em três categorias temáticas: o trabalho do enfermeiro no cuidado de pessoas com lesão de pele, consequências da exposição ao risco ocupacional na realização do cuidado, atuação livre de riscos. Considerações finais: o estudo contribui para que enfermeiros repensem práticas de saúde e segurança do trabalho no cuidado de pessoas com lesão de pele, vislumbrando a saúde e a qualificação do trabalho ao protagonizarem o cuidar.

Descritores: Riscos ocupacionais; Enfermagem; Saúde do trabalhador; Atenção primária à saúde; Ferimentos e lesões.

\section{ABSTRACT}

Objective: to identify in national and international scientific production how the nurse's exposure to occupational risks occurs in the care of the people with skin lesions. Method: integrative review with data collection held in December 2016, in the Portal CAPES, being found publications in the databases PubMed, LILACS e BVS. Results: of the 598 articles found, 33 publications were selected for analysis. The compilation of the articles led to the profile of the publications from 2000 to 2016

1 Enfermeira. Doutora em Enfermagem. Universidade Federal do Rio Grande do Sul. E-mail: dagmar.kaiser@ufrgs.br https://orcid.org/0000-0001-5652-4653

2 Enfermeiro. Instituto de Cardiologia de Porto Alegre, RS. E-mail: roni.1203@outlook.com https: / /orcid.org/0000-0003-0904-2146

3 Enfermeira. Doutora em Enfermagem. Universidade Federal do Rio Grande do Sul. E-mail: carduro@gmail.com https: / /orcid.org/0000-0002-3391-8622

4 Enfermeiro. Doutor em Educação. Universidade Federal do Rio Grande do Sul. E-mail: dilmarpaixao@yahoo.com.br https://orcid.org/0000-0002-6794-8336

5 Enfermeiro. Mestre em Enfermagem. Universidade Federal do Rio Grande do Sul. E-mail: potiguarapaz@yahoo.com.br https://orcid.org/0000-0002-3391-8622 


\section{JOURNALOF

and was derived in three thematic categories: nurses' work in the care of people with lesion, consequences of occupational risk in the performance of care, risk-free performance. Final considerations: the study contributes to nurses rethinking health and safety practices in the care of people with skin lesions, looking at the health and qualification of the work when they carry out the care.

Descriptors: Occupational risks, Nursing; Occupational health; Primary health Care; Wounds and injuries.

\section{RESUMEN}

Objetivo: identificar en la producción científica nacional e internacional como ocurre la exposición del enfermero a los riesgos ocupacionales en el cuidado de personas con lesión de piel. Método: revisión integradora de artículos publicados entre 2000 a 2016, datos recopilados en diciembre de 2016, en el Portal CAPES, encontrándose publicaciones en las bases PubMed, LILACS e BVS. Resultados: 33 de los 598 artículos fueron seleccionados. El compilado de los artículos llevó al perfil de las publicaciones de 2000 a 2016 y derivó en tres categorías temáticas: el trabajo del enfermero en el cuidado de personas con lesión, consecuencias de la exposición al riesgo ocupacional en la realización del cuidado, actuación libre de riesgos. Consideraciones finales: el estudio contribuye a que enfermeros repiensen prácticas de salud y seguridad del trabajo en el cuidado de personas con lesión de piel, vislumbrando la salud y la calificación del trabajo en el cuidado.

Descriptores: Riesgos laborales; Enfermería; Salud laboral; Atención primaria de salud; Heridas y lesiones.

\section{INTRODUÇÃO}

As lesões de pele são responsáveis por uma expressiva parcela dos atendimentos realizados nos diferentes serviços de saúde ${ }^{1}$, deparando-se 0 enfermeiro invariavelmente com as pessoas por elas acometidas ao buscarem por tratamento.

Diante do exposto, ressalta-se a importância da discussão sobre como se dá a exposição do enfermeiro aos riscos ocupacionais enquanto atua no cuidado de pessoas com lesão, ou seja, acometidas por úlceras venosas ou arteriais, lesões por pressão, escoriações, macerações ou queimaduras, deiscências de sutura, estomias, pé diabético, lesões dermatológicas ou decorrentes de amputação, entre outras. ${ }^{1-2}$

São muitos os produtos e tecnologias utilizados no tratamento das lesões, na limpeza dos materiais; inclusive, o enfermeiro, muitas vezes, realiza curativos durante longas jornadas de trabalho ou em espaços físicos impróprios, com insuficiência de medidas protetivas, falta de equipamentos de proteção individual ou a não utilização dos mesmos, somado aos problemas em manter o ambiente criticamente saudável para uma atuação profissional salutar e resolutiva. ${ }^{2-3}$ Reconhecer tais situações e compreender que a exposição ocupacional aos riscos dá-se por meio da interação profissional com a diversidade dos riscos presentes onde o cuidado acontece, permitirá ao enfermeiro desenvolver uma cultura prevencionista e implementar estratégias de intervenção que reduzam ou mesmo eliminem os riscos. ${ }^{4}$

Para tanto, é necessário ao enfermeiro buscar no arcabouço teórico, científico, político, histórico e cultural 0 aporte para a 
 \\ thes}

implementação de medidas efetivas e intervenções que resultem em benefício da segurança e bem-estar no trabalho. Desta forma, ele exercerá suas atividades com o indispensável conjunto de conhecimentos para planejar, tomar decisão e interagir com os riscos ocupacionais com competência e autonomia, bem como preservará a saúde no trabalho no complexo acompanhamento e promoção do cuidado, configurado por um papel humanizado e responsável mediante ações resolutivas às necessidades das pessoas e dos coletivos no cuidado da pele. ${ }^{3-4}$

Ao enfermeiro é necessário, então, ressignificar formas de proteção com base nos riscos ocupacionais presentes no cuidado e às peculiaridades das condições de trabalho envolvidas, com vistas a novos estilos saudáveis de viver e trabalhar enquanto cuida de pessoas com lesão, determinando saúde e segurança no trabalho.

A justificativa para a busca na literatura de um embasamento teórico que responda a essas inquietações está centrada na conscientização da expressiva importância da adoção de práticas seguras pelo enfermeiro no cuidado da pele e em ações de educação em saúde do trabalhador. Igualmente, visa obter elementos para o enfermeiro intervir com vistas à atuação livre de riscos, haja vista as preocupações com as condições de trabalho, o desgaste físico e mental, os acidentes de trabalho e o adoecimento enquanto cuida, observados os limites e parâmetros da legislação vigente $\mathrm{e}^{5-6} \mathrm{e}$ o Código de Ética dos Profissionais de Enfermagem. ${ }^{4}$
ISSN 2236 - 1987

Neste

sentido,

a Norma

Regulamentadora $\mathrm{n}^{\circ} 9$ (NR-9), do Ministério do Trabalho e Emprego ${ }^{5}$, classifica os riscos ocupacionais em: riscos físicos, riscos químicos, riscos biológicos, riscos ergonômicos e riscos de acidentes. Estes riscos reúnem uma complexidade de fatores que podem causar diferentes formas de adoecimento e acidentes a partir do trabalho que precisam ser controlados por práticas seguras de cuidado.

0 enfermeiro tem, no arcabouço legal, garantias que visam à preservação da saúde e redução dos riscos inerentes ao trabalho. A Norma Regulamentadora $n^{\circ} 32$ (NR-32) versa sobre a segurança e saúde no trabalho em serviços de saúde, estabelecendo diretrizes básicas para a implementação de medidas de proteção à segurança e à saúde dos trabalhadores. Destaca, ainda, que sejam desenvolvidas ações de capacitação profissional de forma continuada, orientando quanto aos riscos biológicos, químicos, físicos, ergonômicos e de acidente porventura presentes no trabalho. ${ }^{6}$ Discorre, igualmente, sobre a obrigatoriedade de disponibilização pelo empregador dos Equipamentos de Proteção Individual (EPI) específicos, descartáveis ou não, em número suficiente nos postos de trabalho, garantindo o imediato fornecimento ou reposição. Ao trabalhador, a regulamentação alerta que, em situações de exposição aos riscos ocupacionais, ele deveria permanecer nestas áreas o menor tempo possível para a realização de procedimentos, ter conhecimento dos riscos associados ao seu trabalho, usar os equipamentos de proteção individuais adequados a 


\section{JOURNALOF \\ NURSING \\ aNo HEALTH}

cada tipo de risco e manter acompanhamento em saúde ocupacional. ${ }^{6}$

Dessa forma, buscando respostas à questão norteadora "como acontece a exposição do enfermeiro aos riscos ocupacionais no cuidado de pessoas com lesão de pele?", foi objetivo deste estudo identificar na produção científica nacional e internacional como ocorre a exposição do enfermeiro aos riscos ocupacionais no cuidado de pessoas com lesão de pele.

\section{MÉTODO}

Trata-se de Revisão Integrativa $(\mathrm{RI})^{7}$ que analisou publicações de relevância e suporte à tomada de decisão do enfermeiro à saúde e segurança ocupacional enquanto cuida de pessoas com lesão de pele.

Para a organização da revisão seguiram-se as seguintes etapas: identificação do tema, elaboração da questão norteadora, estabelecimento dos critérios para inclusão e exclusão, categorização dos estudos, análise dos estudos incluídos, interpretação dos resultados e síntese do conhecimento sobre a exposição do enfermeiro aos riscos ocupacionais no cuidado de pessoas com lesão de pele.

A questão norteadora que orientou o problema foi: Como acontece a exposição do enfermeiro aos riscos ocupacionais no cuidado de pessoas com lesão de pele?

Foram critérios de inclusão os artigos nacionais e internacionais de pesquisas publicadas entre os anos de 2000 e 2016, disponibilizados nos idiomas português ou inglês, permitindo acesso online e gratuito do
ISSN 2236 - 1987

texto completo e que reportassem ao risco ocupacional a que está exposto o enfermeiro no cuidado de pessoas com lesão.

Os critérios de exclusão foram publicações de sites de propagandas de produtos para tratamento de pele, artigos duplicados, blogs e vídeos, bem como aquelas que não atendiam à questão norteadora da pesquisa.

Os Descritores em Ciências da Saúde/Medical Subject Headings (DeCs/MeSH) utilizados foram, respectivamente, no idioma português e inglês: Riscos ocupacionais, Occupational Risks; Enfermagem, Nursing; Saúde do trabalhador, Ferimentos e lesões, Wounds and injuries.

A localização dos estudos deu-se por busca online no Portal de Periódicos da Coordenação de Aperfeiçoamento de Pessoal de Nível Superior (CAPES), em dezembro de 2016, encontrando-se publicações disponibilizadas nas bases Publicações Médicas (PubMed), Literatura LatinoAmericana e do Caribe em Ciências da Saúde (LILACS) e Biblioteca Virtual em Saúde (BVS).

Foram feitas todas as combinações possiveis entre os descritores, utilizando-se o conector booleano “AND”. Assim, a busca online disponibilizou 598 artigos. E, de acordo com os juízos de elegibilidade previamente estabelecidos, a amostra final ficou constituída por 33 artigos.

A extração dos dados foi realizada a partir de ponderação criteriosa dos seguintes detalhamentos apontados no roteiro de coleta dos dados: ano de publicação, base de dados, periódico, 
idioma, autores, título do artigo, descritores, tipo de pesquisa, tipo de risco ocupacional e principais resultados encontrados. As publicações foram organizadas em um quadro sinóptico geral, orientando para resultados da pesquisa, atribuindo uma numeração às publicações encontradas.

A análise dos dados ocorreu em duas etapas. Primeiramente foi realizada a caracterização do perfil das publicações conforme os detalhamentos extraídos do roteiro de coleta dos dados. Na segunda etapa procedeu-se a leitura detalhada dos artigos na íntegra e realizou-se análise de conteúdo temática.
ISSN 2236 - 1987

Nesta revisão integrativa é assegurada a autoria dos artigos pesquisados, de forma que todos os estudos utilizados foram referenciados. Por não envolver seres humanos, não foi necessária a aprovação do projeto de pesquisa em Comitê de Ética em Pesquisa.

\section{RESULTADOS}

A amostra final constituiu-se de 33 artigos publicados entre os anos 2000 e 2016. O fluxograma apresentado na Figura 1 detalha os resultados quanto à identificação, seleção e inclusão dos artigos na pesquisa.

Figura 1 - Fluxograma de identificação dos artigos disponibilizados no Portal Capes.

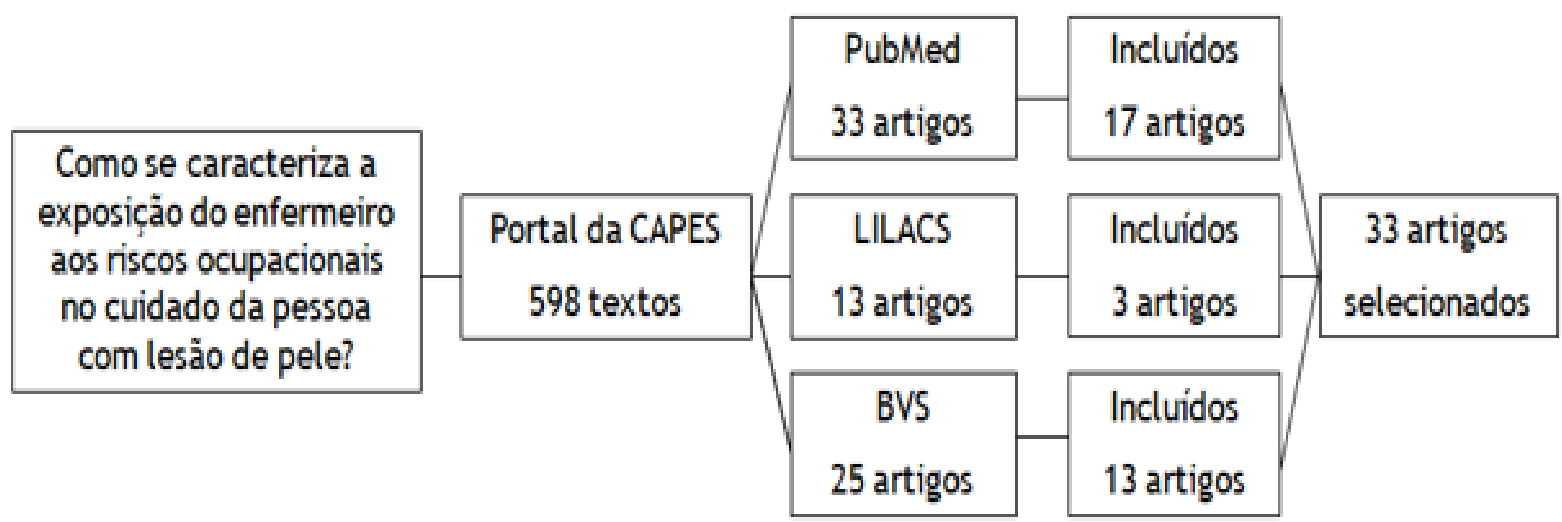

O perfil dos artigos analisados apresentou 19 publicações no idioma

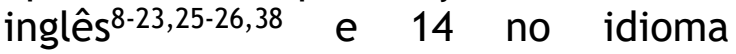
português. ${ }^{24,27-37,39-40}$

Quanto ao tipo de pesquisa empregada nas publicações disponibilizadas na pesquisa destacamse a revisão sistemática, ${ }^{8}$ a pesquisa quantitativa, 10,12-13,16-21,23-25,28-29,31,33,3637 a pesquisa mista quanti- qualitativa ${ }^{35}$, o relato de experiência, ${ }^{39}$ a pesquisa documental, 9,14,26,32,34 a pesquisa qualitativa ${ }^{1,15,27,40}$ e a revisão integrativa. 22,30

Publicações em sites de propagandas de produtos para tratamento de pele, blogs, vídeos, publicações duplicadas ou que não atenderam à questão norteadora da pesquisa foram critérios adotados na 


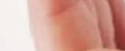

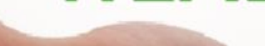

análise que excluíram 565 textos disponibilizados.

O Quadro 1 corresponde aos extratos das publicações analisadas conforme o tipo de risco e exposição ocupacional no cuidado da pele. No entanto, nem sempre os autores reportaram-se especificamente à atuação do enfermeiro no cuidado de pessoas com lesão, e sim, trouxeram contribuições para a área com base em processos de trabalho com participação do enfermeiro em equipe de enfermagem e multiprofissional, na qual ele planejava, organizava, desenvolvia e realizava de forma compartilhada com as equipes o cuidado. Desta forma, os estudos destacaram atividades que estavam sendo executadas quando ocorreu a exposição aos riscos ocupacionais no cuidado da pele, entre elas, o transporte de pacientes e seu manuseio, a administração de medicamentos, a realização e
ISSN 2236 - 1987

procedimentos de enfermagem a pessoas portadoras de vírus, a realização do exame físico durante a consulta de enfermagem, a visita domiciliar, a exposição aos riscos biológico, químico, físico, ergonômico e de acidente durante o trabalho, o trabalho em território a céu aberto, entre outros.

Considerando os resultados encontrados e a questão norteadora "como acontece a exposição do enfermeiro aos riscos ocupacionais no cuidado das pessoas com lesão de pele?", passou-se à leitura detalhada dos artigos na íntegra, visando à análise de conteúdo temática.

A análise do corpus teórico resultou em três categorias temáticas: o trabalho do enfermeiro no cuidado de pessoas com lesão de pele, consequências da exposição ao risco ocupacional na realização do cuidado, atuação livre de riscos; detalhadas a seguir, no Quadro 2.

Quadro 1 - Artigos disponibilizados no portal da CAPES conforme tipo de risco e exposição. Porto Alegre, 2016.

\begin{tabular}{|c|c|c|}
\hline $\mathbf{N}^{0}$ & Tipo de risco & Exposição ocupacional \\
\hline $1^{8}$ & Físico & - Corpo \\
\hline $2^{9}$ & De acidente & - Acidente de trajeto \\
\hline $3^{10}$ & De acidente & - Excessiva produtividade / Stress \\
\hline $4^{11}$ & Biológico & - Reencape de agulhas \\
\hline $5^{12}$ & Ergonômico & $\begin{array}{l}\text { - Arranjos de trabalho inadequados / Transporte de pacientes/Esforço } \\
\text { físico }\end{array}$ \\
\hline $6^{13}$ & Ergonômico & - Manejo inadequado de pacientes \\
\hline $7^{14}$ & Químico & - Queimaduras químicas acidentais no corpo \\
\hline $8^{15}$ & Ergonômico & - Arranjo físico inadequado/Stress \\
\hline $9^{16}$ & Biológico & $\begin{array}{l}\text { - Contato com material biológico/Deficiência de EPI para o trabalho / } \\
\text { Sobrecarga de trabalho }\end{array}$ \\
\hline $10^{17}$ & Biológico & - Contato percutâneo com sangue \\
\hline $11^{18}$ & Biológico & - Vírus da hepatite C / HIV \\
\hline & & Continuação na próxima página. \\
\hline
\end{tabular}


Continuação da página anterior.

$\mathrm{N}^{\mathrm{O}} \quad$ Tipo de Risco

$12^{19}$ Ergonômico - Estressores físicos no trabalho

$13^{20}$ Biológico - Vírus da hepatite A e C

$14^{21}$ Ergonômico - Esforço físico repetitivo

$15^{22}$ Ergonômico - Esforço físico repetitivo

$16^{23}$ Físico - Calor excessivo

$17^{24}$ Biológico - Doenças infecciosas

$18^{25}$ Físico - Ambiente pressurizado

$19^{26}$ Ergonômico - Esforço físico

$20^{27}$ Ergonômico - Arranjo físico inadequado / Dupla jornada de trabalho/Relações interpessoais / Insuficiência de materiais e EPI

$21^{28}$ Todos* - Exposição profissional aos riscos ocupacionais

$22^{29}$ Físico/Químico - Desconhecimento / Exposição constante à luz/Produtos saneantes

$23^{30}$ Todos* - Desconhecimento / Não adesão às precauções padrão

$24^{31}$ Ergonômico - Cargas de trabalho excessivas

$25^{32}$ Riscos velados - Primeira consulta de enfermagem após seis meses a um ano do início da ferida

$26^{33}$ Riscos velados - Excreções / Secreções / Imprevisibilidade

$27^{34}$ Todos* $^{*} \quad$ Conhecimento / Riscos químiconciados pela literatura

$28^{35}$ Todos* $^{*}$ - Conhecimento / Riscos ergonômicos

$29^{36}$ Todos* - Contato com fluidos / Ausência de EPI / Resistência no uso do EPI

$30^{37}$ Biológico - Exposição a material biológico

$31^{38}$ Ergonômico - Vício postural

$32^{39}$ Todos* $^{*} \quad$ Desconhecimento do risco

$33^{40}$ Biológico - Consequências da exposição ocupacional

*Todos: contempla exposição aos riscos biológico, físico, químico, ergonômico e de acidente.

Quadro 2 - Categorias e Subcategorias Temáticas - Porto Alegre, RS, Brasil, 2016.

\begin{tabular}{|c|c|}
\hline Categorias temáticas & Subcategorias temáticas \\
\hline $\begin{array}{l}\text { O trabalho do enfermeiro no cuidado de } \\
\text { pessoas com lesão }\end{array}$ & $\begin{array}{l}\text { - Dinâmica do trabalho }{ }^{10-11,25-26,32-33,35,39-40} \\
\text {-Estabelecimento de protocolos institucionais }{ }^{23,25,27-} \\
28,31,34,36,39 \\
\text {-Equipamentos e insumos }{ }^{19-20,23,28-29-40} \\
\text { - Necessidades das pessoas com lesão } \\
40\end{array}$ \\
\hline $\begin{array}{l}\text { Consequências da exposição ao risco } \\
\text { ocupacional na realização do cuidado }\end{array}$ & $\begin{array}{l}\text {-Necessidades de cuidado profissional em consonância } \\
\text { com os riscos de exposição } 1,8-38-40 \\
\text {-Acidente de trabalho } \\
\text { - } \text { - }^{11,13-14,19,21,31,40} \\
\text { doecimento no trabalho } \\
11,16,19,26,28,35-36,40\end{array}$ \\
\hline Atuação livre de riscos & $\begin{array}{l}\text {-Conhecimento } 9-10,13,23-26,33-36,40 \\
\text {-Precauções universaiss } 8,11,20,25,28,30,35,37,39-40 \\
\text {-Educação permanente } \\
\text {-E-9,11-14,16,20,22-23,25,28-31,36-37,39-40 }\end{array}$ \\
\hline
\end{tabular}




\section{ISSN $2236-1987$}

\section{DISCUSSÃO}

0 trabalho do enfermeiro no cuidado de pessoas com lesão de pele

Mesmo que interligada e complementada por outros saberes profissionais, a Sistematização da Assistência de Enfermagem (SAE) organiza a dinâmica do trabalho da enfermagem, sendo atividade privativa do enfermeiro a realização do histórico de enfermagem, do diagnóstico de enfermagem, do planejamento, a implementação de cuidados e sua avaliação, constituindo uma exigência para as instituições de saúde, tanto públicas como privadas, de todo o Brasil. ${ }^{41}$ No cuidado da pele, a SAE pode ser amplamente contextualizada como a ciência do cuidado integral e integrador em saúde, tanto no sentido de assistir e coordenar as práticas, quanto no sentido de promover e proteger a saúde das pessoas, famílias e comunidades. Neste sentido, o enfermeiro vem buscando conhecimentos teóricos e práticos, além de comportamentais e de atitude para inovar e sustentar as transformações requeridas para este cuidado. ${ }^{10-12,24,26-37,39-40}$

No que tange ao cuidado de pessoas com lesões de pele, a Resolução COFEN $n^{\circ}$ 501/2015² regulamenta as atribuições da equipe de Enfermagem na prevenção e cuidado da ferida com base em quatro estágios distintos: estágio I, com comprometimento da epiderme apenas e a formação de eritema em pele íntegra e sem perda tecidual; estágio II, com abrasão ou úlcera com perda tecidual e comprometimento da epiderme, derme ou ambas; Estágio III, com presença de úlcera profunda e comprometimento total da pele $e$ necrose de tecido subcutâneo, entretanto, a lesão não se estende até a fáscia muscular; Estágio IV, com extensa destruição de tecido, chegando a ocorrer lesão óssea ou muscular ou necrose tissular. ${ }^{2}$

Desse modo, o trabalho do enfermeiro diz respeito a realizar curativos, coordenar e supervisionar a equipe de enfermagem na prevenção e cuidados de feridas, dentre outras atribuições específicas. Ao técnico de enfermagem compete realizar curativos nas feridas de Estágio I e II, em estágio III somente quando delegado pelo enfermeiro, auxiliar o enfermeiro nos curativos de feridas de estágio III e IV, executar as ações prescritas pelo enfermeiro, dentre outras. A atuação do auxiliar de enfermagem prevê a realização de curativos em feridas com estágio I, auxiliar o enfermeiro nos curativos com estágio III e IV, orientar a pessoa quanto aos procedimentos, dentre outros. ${ }^{2}$

Tendo em vista a contextualização da SAE, o cuidado de enfermagem às pessoas com lesão configura-se como prática social empreendedora pela inserção ativa e proativa nos diferentes arranjos de atuação profissional, principalmente, pelas possibilidades interativas e associativas com os diferentes setores e contextos sociais. ${ }^{1,39,41} 0$ enfermeiro torna-se líder e o profissional de referência no contexto do cuidado, na coordenação, na supervisão, na gerência do cuidado e na orientação e 


\section{JOURNALOF \\ NURSING \\ AND HEALTH}

ISSN $2236-1987$

no desenvolvimento dos profissionais de enfermagem.

Nesse sentido, considera-se como aspecto fundamental a assistência sistematizada pelo enfermeiro, pautada em diretrizes que contemplem a avaliação clínica, diagnóstico precoce, planejamento das ações terapêuticas, implementação do plano de cuidados, evolução e reavaliação das condutas, além de trabalho educativo permanente em equipe envolvendo a pessoa, familiares e cuidadores. 1

0 enfermeiro, ao vivenciar situações de trabalho muitas vezes inadequadas, nem sempre as considera perigosas, mesmo conhecendo a presença dos riscos ocupacionais no trabalho ${ }^{10-12,24,26-37,39-40}$, pois acredita que o trabalho terá de ser realizado com vistas à melhora e recuperação das lesões de quem cuida. ${ }^{39}$ No entanto, se faz necessário que ele priorize a preservação de sua saúde na realização do cuidado de pessoas com lesão de pele, reconhecendo a importância da segurança no trabalho, bem como a sua própria segurança, enfatizando nas discussões com a equipe as situações de exposição que decorrem do cuidado. Pode-se citar, como exemplo, o cuidado de si durante a realização de curativos e na monitorização da evolução das lesões e da eficácia do tratamento utilizado, sem deixar de lado o cuidado de si ao longo dessas problematizações.

0 estabelecimento de protocolos institucionais $23,25,27-28,31,34,36,39$ contribui fortemente para obter maior adesão dos profissionais de enfermagem à saúde e segurança no trabalho. ${ }^{40}$ Estes protocolos consistem em estratégia que padroniza as práticas de cuidado com o resgate da valorização profissional, o que certamente incita a adesão às medidas de proteção por estimular uma ação proativa frente aos riscos presentes no trabalho do enfermeiro e equipe. Isto, por si só, reduz a variabilidade de ações de cuidado, melhora a qualificação dos profissionais para a tomada de decisão e facilita a incorporação de novos conhecimentos nos processos de trabalho.

Nesse sentido, a adoção de atitudes proativas na práxis perpassa o trabalho seguro. Para que o enfermeiro possa realizar de forma adequada 0 cuidado e garantir a resolubilidade do serviço é imprescindível que não se coloque em situação de risco. Isso requer conscientização do profissional sobre os riscos ocupacionais presentes no trabalho em uma estrutura física adequada e equipada com insumos para o atendimento da pessoa com lesão de pele. ${ }^{9-10,13,23,26,33-36,40}$ No entanto, nem sempre isso é possível. A depender do serviço onde atua ou lugar em que acontece o cuidado à pessoa com lesão, a inconformidade de equipamentos e insumos compromete a autonomia do enfermeiro para a realização do cuidado integral, uma vez que as normas de segurança e medidas de proteção não são consideradas, 0 que pode gerar insatisfações, desgastes e improvisações na prática do cuidado. 11,20,25,28,30,35,37,39-40

0 trabalho do enfermeiro em prol de melhores práticas de saúde deve considerar as formas como ele se comporta frente aos riscos a que está exposto no cuidado às pessoas com 


\section{JOURNALOF \\ NURSING \\ MOHEALTH}

ISSN 2236 - 1987

peculiaridades que determinam vulnerabilidades no estado de saúde profissional, considerando as necessidades de cuidado das pessoas com lesão de pele nos espaços onde o cuidado acontece, pois a prática profissional está fortemente relacionada à percepção sobre os riscos a que está exposto no trabalho.

Os processos de desgaste do corpo e da mente devem ser considerados importantes e graves, e quando decorrentes das atividades do enfermeiro, implicam em diminuição da capacidade para o trabalho, em adoecimento, em queda da produtividade no trabalho, muitos dias perdidos com afastamentos, além da sobrecarga dos demais profissionais que permanecerem ativos. ${ }^{12,24,26-27,31-}$ $33,38,43$

Exemplificando, a possibilidade de contato do enfermeiro com material biológico como sangue ou outros fluidos orgânicos enquanto cuida das pessoas com lesão é potencialmente capaz de contaminá-lo com agentes biológicos causadores de adoecimento, se não estiver devidamente protegido com luvas, avental e óculos. ${ }^{24} 0$ reconhecimento desses riscos $\mathrm{e}$ as necessidades de cuidado profissional em consonância com os riscos, no cotidiano de trabalho, é fundamental e serve de base para decisões quanto às ações de prevenção, eliminação ou controle dos riscos enquanto cuida.

Igualmente, lembra-se que as condições ambientais e o cuidado realizado nos domicílios, em que as condições sanitárias, muitas vezes, são precárias, impedem o enfermeiro de realizar procedimentos simples como a 


\section{JOURNAIOF

lavagem das mãos, favorecendo a exposição ao risco biológico. ${ }^{34}$

O risco químico também está presente em algumas atividades realizadas pelo enfermeiro no cuidado da pele ao permanecer em contato próximo, diariamente, com uma série de substâncias químicas, como drogas antineoplásicas, antibióticos, desinfetantes, agentes anestésicos, agentes esterilizantes, entre outras. Esses agentes químicos são capazes de provocar intoxicações agudas e crônicas de várias doenças ocupacionais, ,2,28,30,34-36,39 mesmo que a manipulação rotineira dessas substâncias muitas vezes faça com que os enfermeiros não percebam os riscos aos quais se expõem ${ }^{34-35}$, como a possibilidade de inflamabilidade, corrosividade, reatividade e toxicidade. ${ }^{44} \mathrm{~A}$ naturalização dos riscos decorrentes do trabalho é muito perigosa, 0 que torna a conscientização e educação dos profissionais necessária para a prática profissional e sua qualidade de vida.

A exposição ao risco ergonômico ocorre em consequência das adaptações não ergonômicas do próprio lugar onde o enfermeiro realiza o cuidado e, consequentemente, da necessidade de se adaptar à realidade de trabalho, levando-o a adotar, muitas vezes, posturas inadequadas que provavelmente the trarão efeitos maléficos à saúde. 12,24,26-27,31-33,38

Também os esforços de auxílio à pessoa com dificuldade de locomoção e até para se levantar da cama, ou a passagem de macas ao leito e viceversa, as jornadas prolongadas, os horários noturnos, os movimentos repetitivos exigem atenção para um trabalho saudável, na intenção de evitar processos de desgaste diversos, como distúrbios osteomusculares, fadiga, dores em geral e alterações do ritmo circadiano, pelo trabalho noturno. ${ }^{31}$

Temperaturas

extremas, umidade, ruído excessivo ou iluminação artificial predominante são exemplos de risco físico. ${ }^{2,5,25,29}$ As medidas de proteção e segurança são formas de prevenção recomendadas para serem utilizadas pelo enfermeiro enquanto cuida da pessoa com lesão de pele. Assim, para evitar os acidentes de trabalho, fazer uso de EPI constitui uma barreira protetora que reduz efetivamente 0 risco de exposição. 8, 11,20,25,28,30,35,37,39-40

Entretanto, o EPI não elimina o risco ocupacional.

A ocorrência de acidentes, como quedas e escorregadas, dá-se em face das condições do piso ou, no caso dos enfermeiros da atenção básica, em calçadas e escadas irregulares. Ao deslocarem-se pelo território até o domicílio das pessoas com lesão de pele, muitas vezes sem calçamento ou mesmo em locais com mato molhado, terra molhada, buracos, presença de esgoto a céu aberto, pontilhões improvisados, entre outros, os profissionais poderão sofrer acidentes. ${ }^{35}$ No entanto, a razão que pode levar ao acidente é determinada por um conjunto de condições individuais, ambientais e institucionais, sendo o comportamento negligente apenas mais um fator, uma vez que o contexto, as condições coletivas e os recursos disponíveis, bem como a falta de recursos, também 


\section{JOURNALOF \\ NURSING \\ AND 1}

contribuem para os acidentes no trabalho. ${ }^{31}$

\section{Atuação livre de riscos}

Os caminhos que protegem o enfermeiro da exposição aos riscos são determinados por um conjunto de circunstâncias que ele assume no cuidado de pessoas com lesões de pele, pois the compete a tomada de decisão nas ações de maior complexidade da Enfermagem em um cenário cujas informações requerem comunicação, relacionamento interpessoal, consciência de risco e conhecimento da prática profissional. ${ }^{3}$

No conjunto das publicações que compuseram o corpus estudado neste artigo, destacou-se a necessidade de desenvolver a força de trabalho na busca da adoção de práticas seguras. ${ }^{9-}$ 10,13,23-26,33-36 Tais inquietações referem-se, especialmente, à falta de programas de educação que abordem boas práticas de proteção aos riscos ocupacionais e à carência de profissionais qualificados, nessa área, para capacitar trabalhadores. Neste sentido, o cuidado da pele exige conhecimento de normas sobre proteção e segurança no trabalho, dos protocolos de tratamento às pessoas com lesão, dos efeitos e eventos adversos que fundamentam as terapêuticas, visando reduzir a exposição do enfermeiro aos riscos e prevenir acidentes e doenças ocupacionais decorrentes do cuidado.

A atuação do enfermeiro acontece por meio de instrumentos operativos utilizados com a intenção de melhorar as ações do cuidado e requer habilidades e atitudes para 0 alcance da resolutividade com os
ISSN 2236 - 1987

recursos que dispõe para o trabalho, uma vez que mudanças têm sido impulsionadas pela evolução tecnológica de acesso à informação, levando à rápida obsolescência de insumos e equipamentos, suscitando maior proteção na exposição dos profissionais aos riscos ocupacionais e sua prevenção nas peculiaridades do processo de trabalho no cuidado da pele. ${ }^{1-43}$ Para isso, é necessário que haja o estabelecimento e a exigência da documentação do mesmo, contemplando processos que particularizem atribuições e responsabilidades, bem como o monitoramento dos riscos ocupacionais a que está sujeitado o enfermeiro, referentes às suas demandas de trabalho e a sobrecarga de atividades. ${ }^{42}$

A forma como o enfermeiro entende sua realidade profissional corporifica-se em atitudes que reduzem, minimizam ou mesmo extinguem riscos de exposição prejudiciais a sua saúde e integridade física, ou não, independentemente da situação de saúde ou doença e em que acidentes podem ocorrer, requerendo, por vezes, serem modificadas para a realização do cuidado. ${ }^{44}$

Nestas situações, a educação permanente, ou seja, o saber-fazer e o saber ser/agir poderão permitir ao enfermeiro analisar o trabalho que realiza e promover a aprendizagem sobre o seu próprio fazer. ${ }^{10-12,24,26,28-}$ 31,36-37,39-40 Assim, procura-se aproximar conhecimentos de saúde e a segurança no trabalho no contexto do cuidado. 45

0 conhecimento pelo enfermeiro dos riscos a que está exposto, enquanto cuida de pessoas com lesão 


\section{JOURNALOF \\ NURSING \\ AND HEALTH}

de pele, permite inferir que esteja preparado para o enfrentamento e a gestão dos riscos ocupacionais para planejar, implementar e avaliar o cuidado das pessoas com lesão de pele.

\section{CONSIDERAÇÕES FINAIS}

Nas publicações analisadas, a exposição ao esforço físico, os vícios de postura no trabalho, o longo período em que o enfermeiro trabalha em pé no cuidado da ferida, o uso de substâncias químicas como terapêuticas de cuidado da pele ou na limpeza e organização do ambiente, a área geográfica de risco do território e o estresse referente às demandas sociais foram apontadas por exporem o enfermeiro aos riscos ocupacionais no cuidado das pessoas com lesão. No entanto, essa exposição ao risco no trabalho nem sempre foi reconhecida como adoecedora ou com potencial para acidentes, sendo muitas vezes abordada de forma velada.

A discussão dos resultados ressalta que 0 enfermeiro busque tomar decisões enquanto cuida, seja na prevenção ou na cura, com atitudes embasadas no conhecimento científico e em práticas seguras de cuidado, que considerem a problemática de exposição aos riscos no cuidado da pele, haja vista o direito à saúde no trabalho e as repercussões na saúde ocupacional.

Além disso, percebe-se a organização do trabalho como um processo que permeia o modo de ser e de viver do enfermeiro, está aí um campo fértil para investimento em pesquisas, na medida em que a exposição aos riscos ocupacionais incita mudanças nos condicionantes da
ISSN 2236 - 1987

saúde ocupacional e do processo de trabalho do enfermeiro no cuidado da pele. Permitir acesso à informação e as inovações na prática profissional podem se tornar uma efetiva proteção aos riscos ocupacionais, sendo que a gestão dos serviços de saúde pode oportunizar espaços pedagógicos e promover novos arranjos na organização do cuidado, contribuindo, deveras, para a prevenção de agravos à saúde na realização do cuidado.

Importante ressaltar que os estudos sobre os riscos ocupacionais analisados se deram em distintas realidades de atuação do enfermeiro. Contudo, o que se observou é que tais estudos se detêm em investigar informações que os profissionais têm acerca dos riscos ocupacionais ou em ponderar o uso, ou não, dos equipamentos de proteção individual. No entanto, não foram encontrados na literatura mundial estudos que avançassem com propostas de práticas seguras considerando a exposição ao risco ocupacional enquanto cuida de pessoas com lesão ou no campo do cuidado da pele. Essas discrepâncias representam as lacunas presentes na atuação do enfermeiro que envolvem a saúde ocupacional, com a finalidade de embasar a dinâmica de trabalho no cuidado de pessoas com lesão de pele, bem como da necessidade de cuidado para preservar sua saúde.

A educação permanente surge como alternativa para promover a informação, a conscientização e a educação em serviço e em saúde para um trabalho livre de riscos, visando trazer proteção e segurança ao contexto de trabalho, bem como à proteção dos direitos profissionais do 
enfermeiro e, por extensão, aos demais integrantes da equipe de trabalho. São destacadas as rodas de conversa e a discussão de casos, atividades que poderão agregar elementos que qualifiquem a conduta e transformem comportamentos muitas vezes inadequados.

Desta forma, este estudo agrega conhecimento científico à área de Saúde do Trabalhador por apresentar dados importantes sobre a exposição de enfermeiros aos riscos ocupacionais no cuidado da pele. Esse compilado de informações apresenta subsídios significativos e consistentes para 0 planejamento do cuidado e do ensino de Enfermagem com vistas a um desempenho profissional competente do enfermeiro, alerta para as instituições formadoras e os serviços de saúde.

Quanto às limitações, possíveis viés e/ou fragilidades do estudo, nem sempre nas publicações foram problematizadas, detalhadamente, as práticas seguras pelo enfermeiro na realização do cuidado de pessoas com lesão de pele para suplantar as possíveis situações de risco. Destacase, ainda, o fato de que foram incluídos no estudo apenas artigos disponíveis online e gratuitos, desconsiderando outras formas de divulgação.

Encerra-se este artigo com 0 desejo de contribuir para enfermeiros repensarem práticas, identificarem potencialidades e fragilidades no cuidado às pessoas com lesão de pele e na organização do trabalho que realizam, trazendo, inclusive, segurança às suas ações, com valorização das equipes e 0 seu empoderamento, vislumbrando a saúde e a qualificação do trabalho à medida que o enfermeiro se percebe como protagonista no processo de cuidar.

\section{REFERÊNCIAS}

1 Malagutti W, Kakihara CT. Curativos, estomias e dermatologias: uma abordagem multiprofissional. $3^{a}$ ed. São Paulo: Martinari; 2014.

2 Conselho Federal de Enfermagem (COFEN). Resolução $n^{\circ}$ 0501/2015: regulamenta a competência da equipe de enfermagem no cuidado às feridas e dá outras providências. Brasília; 2015.

3 Loro MM, Zeitoune RCG. Collective strategy for facing occupational risks of a nursing team. Rev esc enferm USP [Internet]. 2017 [cited 2017 Apr 04];51:e03205. Available from: http://www.scielo.br/scielo.php?scrip $\mathrm{t}=\mathrm{sci}$ _arttext\&pid=S0080-

62342017000100402\&lng=pt

4 Conselho Federal de Enfermagem (COFEN). Resolução $n^{\circ}$ 564/2017: aprova o novo código de ética dos profissionais de enfermagem. Brasília; 2017.

5 Ministério da Saúde (BR). Portaria $\mathrm{n}^{\circ}$ 25, de 29 de dezembro de 1994: apresenta Norma Regulamentadora 9 programa de prevenção de riscos ambientais. Brasília; 1994.

6 Ministério do Trabalho e Emprego (BR). Portaria $n^{\circ}$ 485, de 11 de novembro de 2005: aprova a norma regulamentadora $n^{\circ} 32$ - segurança e saúde no trabalho em estabelecimentos de saúde. Brasília; 2005. 


\section{ISSN 2236 - 1987}

7 Mendes KDS, Silveira RCCP, Galvão CM. Revisão integrativa: método de pesquisa para incorporação de evidências em saúde e na enfermagem. Texto \& contexto enferm. [Internet].2008 [acesso em 2018 jul 28];7(4):758-64. Disponível em: http://www.scielo.br/pdf/tce/v17n4/ 18.pdf

8 Kesavachandran CN, Haamann F, Nienhaus A. Radiation exposure of eyes, thyroid gland and hands in orthopaedic staff: a systematic review. Eur $\mathrm{j}$ med res. [Internet].2012 Out [cited 2016 Sept 28];17(1):28. Available from: http://dx.doi.org/10.1186/2047783X-17-28

9 Psoinos CM, Emhoff TA, Sweeney WB, Tseng JF, Santry HP. The dangers of being a "weekend warrior": A new call for injury prevention efforts. J trauma acute care surg. [Internet].2012 Aug [cited 2016 Sept 28];73(2):469-73. Available from: http://dx.doi.org/10.1097/TA.0b013e $318258437 c$

10 Marinescu LG. Integrated approach for managing health risks at work-the role of occupational health nurses. AAOHN j [Internet]. 2007 Feb [cited 2016 Sept 28];55(2):75-87. Available from:

http://journals.sagepub.com/doi/abs /10.1177/216507990705500205?journa ICode=whsc

11 Nawafleh H, Francis KL, Chapman YB. The influence of HIV/AIDS on the practice of primary care nurses in Jordan: rhetoric and reality. Int j nurs pract [Internet]. 2005 [cited $2016 \mathrm{Sept}$ 28];11(5):200-5. Available from: http://dx.doi.org/10.1111/j.1440172X.2005.00528.X

12 Brown JG, Trinkoff A, Rempher K, McPhaul K, Brady B, Lipscomb J, et al. Nurses' inclination to report workrelated Injuries: organizational, workgroup, and individual factors associated with reporting. AAOHN j [Internet]. 2005 May[cited 2016 Sept 28];53(5):213-7. Available from: http:// journals.sagepub.com/doi/abs /10.1177/216507990505300505?journa ICode=whsc

13 Daynard D, Yassi A, Cooper JE, Tate R, Norman R. Wells R. Biomechanical analysis of peak and cumulative spinal loads during simulated patienthandling activities: a substudy of a randomized controlled trial to prevent lift and transfer injury of health care workers. Appl ergon [Internet]. 2001[cited 2016 Sept 28];32(3):199214. Available from: https://www.sciencedirect.com/scien ce/article/pii/S0003687000000703?via \%3Dihub

14 Adepoju F G, Adeboye A, Adigun I A. Chemical eye injuries: Presentation and management difficulties. Ann afr med [Internet]. 2007 [cited 2016 Sept 22];6(1):7-11. Available from: http://www. annalsafrmed.org/text.a sp?2007/6/1/7/55738

15 D'Arcy LP, Sasai Y, Stearns SC. Do assistive devices, training, and workload affect injury incidence? Prevention efforts by nursing homes and back injuries among nursing assistants. J adv nurs [Internet]. 2012 [cited 2016 Oct 28];68(4):836-45. Available from: http://dx.doi.org/10.1111/j.13652648.2011.05785.x 
16 Santos JLG, Vieira M, Assuiti LFC, Gomes D, Meirelles BHS, Santos SMA. Risco e vulnerabilidade nas práticas dos profissionais de saúde. Rev gauch enferm [Internet]. 2012 jun [acesso em 2016 out 22];33(2):205-12. Disponível em: http://dx.doi.org/10.1590/S198314472012000200028

17 Leiss JK. Management practices and risk of occupational blood exposure in U.S. paramedics: non-intact skin exposure. Ann epidemiol [Internet]. 2009 [cited 2016 Oct 30];19(12):88490. Available from: http://dx.doi.org/10.1016/j.annepide m.2009.08.006

18 Wu HC, Ho JJ, Lin MH, Chen CJ, Guo $\mathrm{YL}$, Shiao JS. Incidence of percutaneous injury in Taiwan healthcare workers. Epidemiol infect [Internet]. 2015 Nov[cited 2016 Sept 28];143(15):3308-15. Disponível em: http://dx.doi.org/10.1017/S09502688 15000321

19 Grayson D, Dale AM, Bohr P, Wolf L, Evanoff B. Ergonomic evaluation part of a treatment protocol for musculoskeletal injuries. AAOHN j. 2005;53(10):450-7.

20 Kosgeroglu N, Ayranci U, Vardareli $E$, Dincer S. Occupational exposure to hepatitis infection among Turkish nurses: frequency of needle exposure, sharps injuries and vaccination. Epidemiol infect [Internet]. 2004 Feb [cited 2016 Nov 27];132(1):27-33. Available from: http: / /dx.doi.org/10.1017/S09502688 03001407

21 Fragala G, Bailey LP. Addressing occupational strains and sprains: musculoskeletal injuries in hospitals. AAOHN j. 2003 jun;51(6):252-9.
22 Hefti KS, Farnham RJ, Docken L, Bentaas R, Bossman S, Schaefer J. Back injury prevention: a lift team success story. AAOHN j. 2003 jun;51(6):246-51.

23 Arbury S, Jacklitsch B, Farquah O, Hodgson M, Lamson $\mathrm{G}$, Martin $\mathrm{H}$, et al. Heat illness and death among workers - United States, 2012-2013. MMWR morb mortal wkly rep. 2014 ago;63(31):661-5.

24 Cardoso ACM, Figueiredo RM. Situações de risco biológico presentes na assistência de enfermagem nas unidades de saúde da família (USF). Rev latino-am enfermagem [Internet]. 2010 jun [acesso em 2016 nov 22];18(3):368-72. Disponível em: http://dx.doi.org/10.1590/S010411692010000300011

25 Hansen $M B$, Jansen $T$, Sifakis $M B$, Hyldegaard $O$, Jansen EC. Chamber personnel's use of Nitrox 50 during hyperbaric oxygen treatment: a quality study-research report. Undersea hyperb med. 2013 Sept/Oct;40(5):395402.

26 Yassi A, Lockhart K. Workrelatedness of low back pain in nursing personnel: a systematic review. Int $j$ occup environ health [Internet]. 2013 July/Sept [cited 2016 Sept 28];19(3):223-44. Available from: http://dx.doi.org/10.1179/204939671 3Y.0000000027

27 Fontana RT, Lautert L. A situação de trabalho da enfermagem e os riscos ocupacionais na perspectiva da ergologia. Rev latino-am enfermagem [Internet]. 2013[acesso em 2016 out 22];21(6):1306-13. Disponível em: http://dx.doi.org/10.1590/01041169.3105.2368 
28 Silva RR, Bezerra ALD, Sousa MNA. 0 trabalho de enfermagem na hemodiálise: uma abordagem sobre os riscos ocupacionais. C\&D-Revista eletrônica da FAINOR [Internet]. 2012 jan/dez [acesso em 2016 set 22];5(1):101-13. Disponível em: http://srv02.fainor.com.br/revista/in dex.php/memorias/article/view/152/ 121

29 Sulzbacher E, Fontana RT. Concepções da equipe de enfermagem sobre a exposição a riscos físicos e químicos no ambiente hospitalar. Rev bras enferm [Internet]. $2013 \mathrm{fev}$ [acesso em 2016 set 22];66(1):25-30. Disponível em: http://dx.doi.org/10.1590/S003471672013000100004

30 Silva LCP, Juliani CMCM. Biossegurança e risco ocupacional na atenção primária: revisão integrativa da literatura. Rev univ vale Rio Verde [Internet]. 2014 jan/jul[acesso em 2016 set 22];12(1):262-81. Disponível em:

http://periodicos.unincor.br/index.ph $\mathrm{p} /$ revistaunincor/article/view/1355/p df_112

31 Felli VEA, Costa TF, Baptista PCP, Guimarães ALO, Anginoni BM. Exposição dos trabalhadores de enfermagem às cargas de trabalho e suas consequências. Rev esc enferm USP [Internet]. $2015 \mathrm{dez}$ [acesso em 2016 set 22];49(n.esp.):98-105. Disponível em: http://dx.doi.org/10.1590/S0080623420150000800014

32 Liedke DCF, Johann DA, Danski MTR. Consultório de enfermagem para tratamento de feridas em hospital de ensino. Cogitare enferm [Internet].
$2014 \mathrm{jul} / \mathrm{set}$ [acesso em 2016 set 22];19(3):590-6. Disponível em: https://revistas.ufpr.br/cogitare/arti cle/viewFile/34486/23254

33 Baratieri T, Sangaleti CT, Trincaus MR. Conhecimento de academicos de enfermagem sobre avaliação e tratamento de feridas. Rev enferm atenção saude[Internet]. 2015 jan/jun [acesso em 2016 set 22];4(1):2-15. Disponível em: http://seer.uftm.edu.br/revistaeletro nica/index.php/enfer/article/view/12 $59 / 1130$

34 Bessa MEP, Almeida MI, Araújo MFM, Silva MJ. Riscos ocupacionais do enfermeiro atuante na estratégia de saúde da família [Internet]. Rev enferm UERJ. 2010 out/dez[acesso em 2016 set 22];18(4):644-9. Disponível em:

http://www.facenf.uerj.br/v18n4/v1 8n4a24.pdf

35 Nunes MBG, Robazzi MLCC, Terra FS, Mauro MYC, Zeitone RCG, Secco IAO. Riscos ocupacionais dos enfermeiros atuantes na atenção à saúde da família. Rev enferm UERJ [Internet]. $2010 \mathrm{abr} /$ jun[acesso em 2016 set 22];18(2):204-9. Disponível em:

http://www.facenf.uerj.br/v18n2/v1 8n2a07.pdf

36 Rodrigues LMC, Silva CCS, Silva VKBA, Martiniano CS, Silva ACO, Martins MO. Riscos Ocupacionais: percepção de profissionais de enfermagem da Estratégia Saúde da Família em João Pessoa - PB [Internet]. Rev bras cienc saude. 2012[acesso em 2016 set 22];16(3):325-32. Disponível em: 
http://periodicos.ufpb.br/index.php/ rbcs/article/viewFile/12660/7871

37 Malaguti-Toffano SE, Santos CB, Canini SRMS, Galvão MTG, Brevidelli MM, Gir E. Adesão às precauçõespadrão de profissionais de enfermagem de um hospital universitário. Acta paul enferm [Internet]. 2012 [acesso em 2016 nov 22];25(3):401-7. Disponível em: http://dx.doi.org/10.1590/S010321002012000300013

38 Younga JG, Trudeaua M, Odellb D, Marinellib K, Dennerlein JT. Touchscreen tablet user configurations and case-supported tilt affect head and neck flexion angles. Work [Internet]. 2012 [cited 2016 Oct 22];41(81):81-91. Available

from: http://dx.doi.org/10.3233/WOR-20121337

39 Macedo MML, Rodrigues RN, Cortez DN, Lanza FM, Gontijo TL. Abordagem ao portador de úlceras crônicas no município de Divinópolis-MG. Rev APS[Internet]. 2013 out/dez[acesso em 2016 set 22];16(4):474-8. Disponível em:

https://aps.ufjf.emnuvens.com.br/ap s/article/view/1902/772

40 Marziale MHP, Santos HEC, Cenzi CM, Rocha FLR, Trovó MEM. Consequências da exposição ocupacional a material biológico entre trabalhadores de um hospital universitário. Esc anna nery[Internet]. 2014[acesso em 2016 set 22];18(1):11$6 . \quad$ Disponível em: http://www.scielo.br/pdf/ean/v18n1 /1414-8145-ean-18-01-0011.pdf

41 Silva JP, Garanhani ML, Peres AM. Sistematização da assistência de enfermagem na graduação: um olhar sob o pensamento complexo. Rev latino-am enfermagem [Internet]. 2015[acesso em 2017 set 09];23(1):59$66 . \quad$ Disponível em: http:/ /dx.doi.org/10.1590/01041169.0096.2525

42 Domansky RC. Elaboração de protocolos. In: Domansky RC, Borges EL. Manual para prevenção de lesões de pele: recomendações baseadas em evidências. $2^{\mathrm{a}}$ ed. Rio de Janeiro: Rubio; 2014.

43 Siman AG, Brito MJM. Mudanças na prática de enfermagem para melhorar a segurança do paciente. Rev gauch enferm [Internet]. 2016 [acesso 2017 set 10];37(n.esp.):e68271. Disponível em: http://dx.doi.org/10.1590/19831447.2016.esp.68271

44 Costa TF, Felli VEA, Baptista PCP. Nursing workers' perceptions regarding the handling of hazardous chemical waste. Rev esc enferm USP[Internet]. 2012 [cited 2016 sept 22];46(6):1453$61 . \quad$ Disponível em: http://www.scielo.br/pdf/reeusp/v46 n6/en_24.pdf

45 Sena RR, Grillo MJC, Pereira LA, Belga SMMF, França BD, Freitas CP. Educação permanente nos serviços de saúde: atividades educativas desenvolvidas no estado de Minas Gerais, Brasil. Rev gauch enferm. [Internet] 2017 [acesso em 2018 jul 28];38(2):e64031. Disponível em: http:// www.scielo.br/pdf/rgenf/v38n 2/0102-6933-rgenf-1983144720170264031.pdf

Data de submissão: 28/05/2017

Data de aceite: 27/06/2018

Data de publicação: 28/09/2018 